\title{
Dual-modal cancer detection based on optical pH sensing and Raman spectroscopy
}

Soogeun Kim

Seung Ho Lee

Sun Young Min

Kyung Min Byun

Soo Yeol Lee 


\title{
Dual-modal cancer detection based on optical pH sensing and Raman spectroscopy
}

\author{
Soogeun Kim, ${ }^{a}$ Seung Ho Lee, ${ }^{b}$ Sun Young Min, ${ }^{c}$ Kyung Min Byun, ${ }^{a, *}$ and Soo Yeol Lee ${ }^{a, *}$ \\ ${ }^{a} K y u n g$ Hee University, Department of Biomedical Engineering, Yongin, Republic of Korea \\ ${ }^{b}$ Kyung Hee University, Department of Medical Engineering, Seoul, Republic of Korea \\ 'Kyung Hee University School of Medicine, Department of Surgery, Seoul, Republic of Korea
}

\begin{abstract}
A dual-modal approach using Raman spectroscopy and optical pH sensing was investigated to discriminate between normal and cancerous tissues. Raman spectroscopy has demonstrated the potential for in vivo cancer detection. However, Raman spectroscopy has suffered from strong fluorescence background of biological samples and subtle spectral differences between normal and disease tissues. To overcome those issues, $\mathrm{pH}$ sensing is adopted to Raman spectroscopy as a dual-modal approach. Based on the fact that the $\mathrm{pH}$ level in cancerous tissues is lower than that in normal tissues due to insufficient vasculature formation, the dual-modal approach combining the chemical information of Raman spectrum and the metabolic information of $\mathrm{pH}$ level can improve the specificity of cancer diagnosis. From human breast tissue samples, Raman spectra and $\mathrm{pH}$ levels are measured using fiber-optic-based Raman and $\mathrm{pH}$ probes, respectively. The $\mathrm{pH}$ sensing is based on the dependence of $\mathrm{pH}$ level on optical transmission spectrum. Multivariate statistical analysis is performed to evaluate the classification capability of the dual-modal method. The analytical results show that the dual-modal method based on Raman spectroscopy and optical pH sensing can improve the performance of cancer classification. ๑ 2017 Society of Photo-Optical Instrumentation Engineers (SPIE) [DOI: 10.1117/1.JBO.22.10.105002]
\end{abstract}

Keywords: Raman spectroscopy; pH sensing; dual-modal; cancer detection; diagnosis; principal component linear discriminant analysis.

Paper 170349PRR received May 31, 2017; accepted for publication Sep. 22, 2017; published online Oct. 12, 2017.

\section{Introduction}

Over the last decades, Raman spectroscopy has been actively applied to cancer diagnosis due to its capability to detect a slight variation of chemical composition in biological tissue, caused by malignant transformation. Previous studies have shown that Raman spectroscopy can discriminate between normal and cancerous tissue in various tissues, such as cervix, ${ }^{1-3}$ skin,,${ }^{4-7}$ breast, ${ }^{8,9}$ and gastrointestinal tract. ${ }^{10-13}$ However, Raman spectroscopy has suffered from strong fluorescence background in biological applications. In addition, the subtle spectral differences between normal and disease tissues make it difficult to analyze the Raman spectra. To overcome those issues, many researchers have proposed the hardware- and/or software-based methods, such as surface-enhanced Raman scattering ${ }^{2,14}$ and data preprocessing. ${ }^{15,16}$

While the suggestions for improving the quality of Raman spectra received the most attention in previous studies, a multimodal approach, which combines Raman spectroscopy with other methods, was also of interest to researchers. ${ }^{17-19}$ Because the malignant transformation of tissues leads to the change in metabolism and optical characteristics as well as in the chemical composition of the tissues, it is expected that we could improve the accuracy and reliability of cancer diagnosis by merging the tissue state data obtained from multimodal methods. For example, Ashok et al. ${ }^{17}$ reported that both sensitivity of 0.89 and specificity of 0.77 were improved to 0.94 when combining Raman spectroscopy and optical coherence tomography. Lim

*Address all correspondence to: Kyung Min Byun, E-mail: kmbyun@khu.ac.kr; Soo Yeol Lee, E-mail: sylee01@ khu.ac.kr et al. ${ }^{18}$ found that a combination of three fiber-optic-based optical spectroscopy modalities of diffuse optical spectroscopy, laser-induced fluorescence spectroscopy, and Raman spectroscopy showed enhanced performance for in vivo noninvasive diagnosis of melanoma and nonmelanoma skin cancer. Kenny et al. ${ }^{19}$ demonstrated the potential of multimodal spectra imaging based on autofluorescence imaging and Raman microspectroscopy for realizing fast diagnosis of tumors at the surface of large surgical resections with no tissue preparation, such as sectioning and staining.

Commonly, it has been known that tumor microenvironment is hypoxic and acidic owing to the chaotic and heterogeneous microvascular structure of solid tumors. ${ }^{20}$ The $\mathrm{pH}$ level of the extracellular matrix around cancer cells has been reported to be in the range of 5.5 to $6.5 .^{21,22}$ Negrini et al. ${ }^{22}$ revealed that the $\mathrm{pH}$ difference in tissue is attributed to an insufficient vasculature to remove the acidic byproducts of increased anaerobic cellular activity of the rapidly proliferating tumor cells, resulting in lower extracellular $\mathrm{pH}$ of tumors compared to contiguous tissues. Therefore, tissue $\mathrm{pH}$ level can provide useful and complementary information about cellular metabolism of the tissue in cancer diagnosis using Raman spectroscopy.

In this study, we intend to present the dual-modal approach for breast cancer diagnosis based on Raman spectroscopy and optical $\mathrm{pH}$ sensing. The chemical and metabolic variations in normal and cancerous breast tissue samples are measured using fiber-optic-based Raman and $\mathrm{pH}$ probes, respectively. For dualmodal approach, the Raman data are combined with the $\mathrm{pH}$ sensing data. All measured Raman data and combined data

1083-3668/2017/\$25.00 @ 2017 SPIE 
are used to perform the classification analysis with principal component linear discriminant analysis (PC-LDA) followed by leave-one-out cross validation (LOOCV). From the results of the sensitivity and specificity obtained from the classification analysis, it is demonstrated that the dual-modal approach improves the classification capability and has the potential for more accurate breast cancer detection.

\section{Materials and Methods}

\subsection{Sample Preparation}

This study was approved by the Ethics Committee of Kyung Hee University Medical Center (KMC IRB 2016-04-103-003) and all participants signed an informed consent. Twenty breast tissue samples were collected from five participants during breast surgery. Ten samples were from the malignant tumor mass (cancer core area) and the others were from the safety margin outside of the tumor mass. Histological analysis of each sample was performed by expert pathologists and its result was used as gold standard to evaluate the performance of the proposed dual-modal cancer detection. All samples were snap frozen in liquid nitrogen after the collection and stored at $-80^{\circ} \mathrm{C}$ to keep their metabolic content and chemical composition as in vivo state. ${ }^{13,23}$ Just before Raman and optical $\mathrm{pH}$ sensing experiments, each sample was thawed for about $10 \mathrm{~min}$ at room temperature. The size of each sample was about $5 \mathrm{~mm} \times 5 \mathrm{~mm} \times 1 \mathrm{~mm}$.

\subsection{Raman Spectroscopy}

The Raman lensed fiber-optic probe ${ }^{24}$ (Raman probe, EmVision LLC) was used to obtain the Raman spectra from the breast tissue samples as shown in Fig. 1. The Raman probe consists of one central excitation fiber and seven Raman signal collection fibers, which have a low hydroxyl $(\mathrm{OH})$ content, 300- $\mu$ m core diameter and 0.22 NA. For the sample end of the Raman probe, a bandpass filter and a donut-shaped longpass filter are positioned in front of the central excitation fiber and the seven Raman signal collection fibers, respectively, to remove the excitation source signal and pass the Stokes Raman signal. The excitation source of $785 \mathrm{~nm}$ (I0785MM0350MF, Innovative Photonic Solutions) is delivered on the sample surface with the laser power of $200 \mathrm{~mW}$ through the central excitation fiber. Then, the Raman scattering signal reflected from the

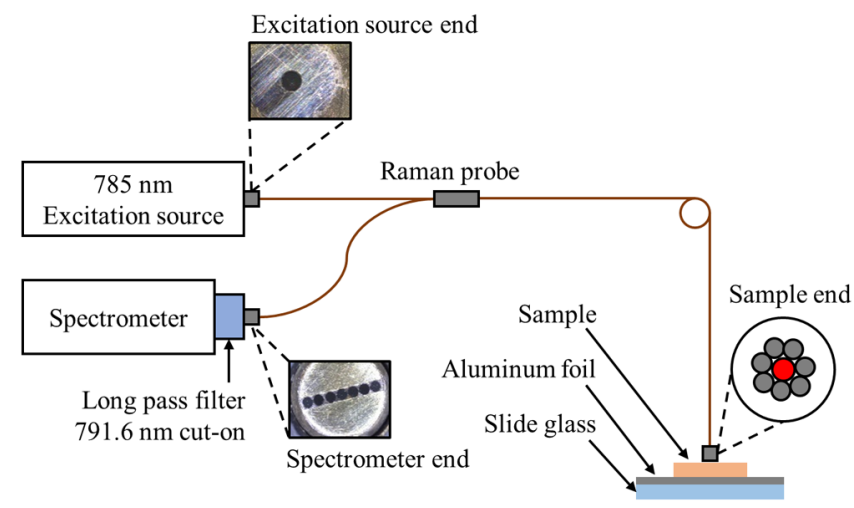

Fig. 1 Schematic of fiber-optic-based Raman spectroscopy for biological sample. The fiber-optic-based Raman probe consists of one central excitation fiber and seven Raman signal collection fibers (see sample end). sample surface is collected and delivered through the seven Raman signal collection fibers and detected by a CzernyTurner spectrograph (SR-303i-A, Andor Technology) with a low dark current deep-depletion CCD (iVac, Andor Technology).

For Raman experiments, each thawed sample was laid on a slide glass surrounded by aluminum foil. The strong and broad fluorescence signal of a slide glass caused by a 785-nm source is prevented by aluminum foil because of its infrared opacity. ${ }^{25} \mathrm{In}$ addition, aluminum foil yields a very low and featureless spectral background, which enables the acquisition of high-quality Raman spectra from biological samples. ${ }^{25}$ Twenty Raman spectra from two different positions were measured for each breast tissue sample. All Raman spectra were acquired in the range of 600 to $1800 \mathrm{~cm}^{-1}$ with the spectral resolution of $0.8 \mathrm{~cm}^{-1}$ and the acquisition time of $1 \mathrm{~s}$.

\subsection{Optical $p H$ Sensing}

To optically measure the $\mathrm{pH}$ variation between normal and cancerous samples, a fiber-optic-based $\mathrm{pH}$ probe was made by the layer-by-layer electrostatic self-assembly technique. ${ }^{26} 3$-Amino7-dimethylamino-2-methylphenazine hydrochloride, commonly known as neutral red (NR), and poly(acrylic acid) (PAA, $\left.M_{w} \sim 1800\right)$ were used as the cationic chemical and anionic polyelectrolyte, respectively, for the fabrication of the nanostructured sensitive films. Ten millimolar solutions of the two chemicals was prepared using deionized water. Then, the $\mathrm{pH}$ of the solutions was adjusted to $\mathrm{pH} 4.2$ by adding a few drops of $\mathrm{NaOH}$ or $\mathrm{HCl}$ to achieve the strong and stable electrostatic interactions between the two chemicals. ${ }^{27}$ All chemicals were purchased from Sigma-Aldrich Inc. and used without further purification.

A multimode plastic-clad silica-core fiber (FT200EMT, Thorlabs Inc., $200-\mu \mathrm{m}$ core diameter, $225-\mu \mathrm{m}$ cladding diameter, $0.39 \mathrm{NA}$ ) was used to fabricate the $\mathrm{pH}$ probe. About 1$\mathrm{cm}$ part of the optical fiber cladding was chemically removed with acetone prior to the polymeric coating fabrication. The exposed part was first immersed into $1 \mathrm{M} \mathrm{KOH}$ solution for $10 \mathrm{~min}$ to acquire negative charge at the surface of the optical fiber core and then was rinsed in deionized water. Afterward, the exposed part was immersed into the $10 \mathrm{mM}$ NR solution for 2 min to fabricate the cationic layer and rinsed in deionized water for $1 \mathrm{~min}$. After the exposed part was immersed into $10 \mathrm{mM}$ PAA solution for $2 \mathrm{~min}$ to fabricate the anionic layer and rinsed again in deionized water for $1 \mathrm{~min}$. From this sequence, the bilayer of NR/PAA was fabricated at the exposed part of the optical fiber. In this study, the sequence was repeated 15 times. The transmission characteristics of the fabricated $\mathrm{pH}$ probe were investigated in the range of 640 to $760 \mathrm{~nm}$ using the standard buffer solutions of $\mathrm{pH}$ values 6,7 , and 8 . To obtain the transmission spectra of Fig. 2(a), whenever each standard $\mathrm{pH}$ solution was dropped onto the surface of the exposed part of the fiber-optic-based $\mathrm{pH}$ probe ( $\mathrm{pH}$-sensitive region), the transmission spectra at $\mathrm{pH}$ values 6,7 , and 8 were measured by the spectrograph with CCD used in Raman experiment (refer to Fig. 3). The observed variation trend of the transmission spectra according to $\mathrm{pH}$ level is consistent with earlier study. ${ }^{28}$ Figure 2(b) shows the calibration curve of the $\mathrm{pH}$ probe between $\mathrm{pH}$ values 6 and 8 , which was obtained using the integrated transmission intensity values, areas under the transmission spectral curves of Fig. 2(a). 

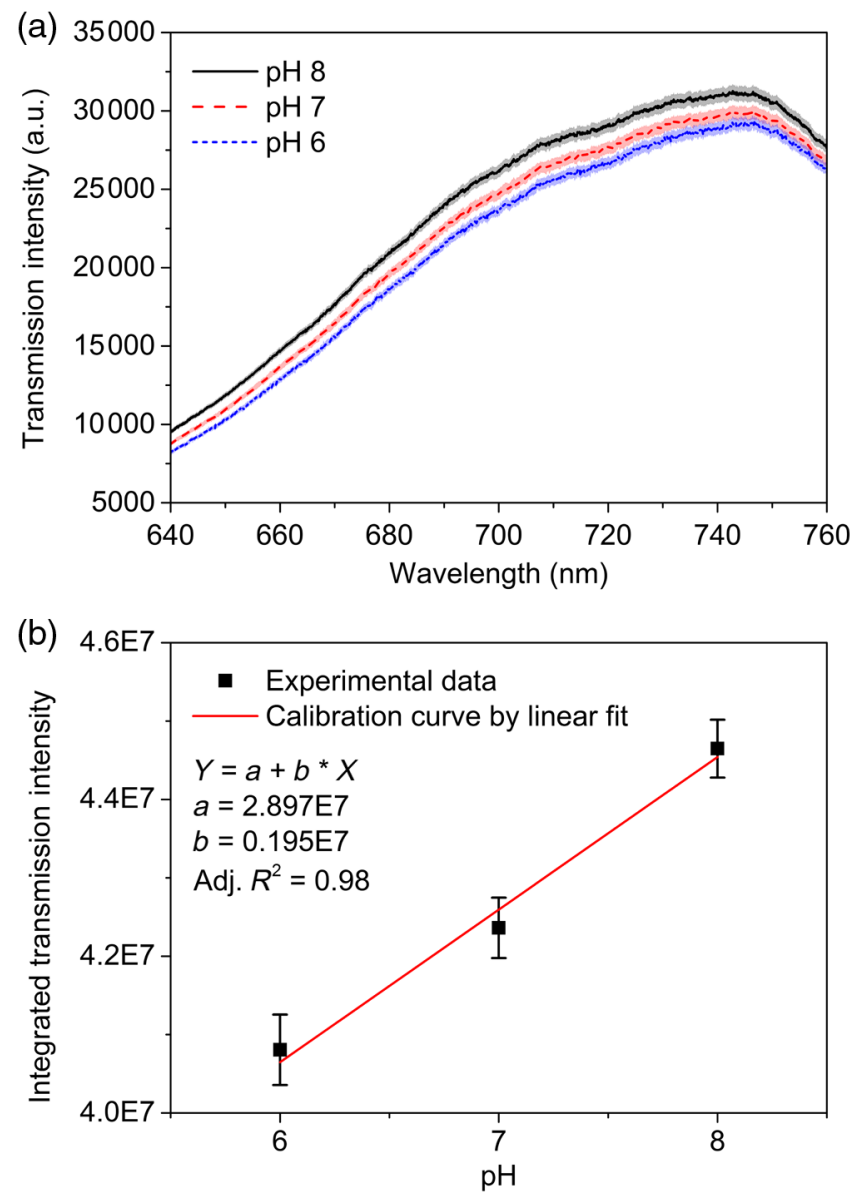

Fig. 2 (a) Transmission spectra measured at the $\mathrm{pH}$ values 6,7 , and 8 of standard buffer solutions by the optical $\mathrm{pH}$ probe (solid and dashed lines: average and filled areas: standard deviation) and (b) calibration curve for the optical $\mathrm{pH}$ probe estimated using the integrated transmission intensity values [areas under the transmission spectral curves of Fig. 2(a)] at the $\mathrm{pH}$ values 6,7 , and 8.

After the calibration of the $\mathrm{pH}$ probe, the transmission spectra of the normal and cancerous breast tissue samples were also measured immediately after Raman experiments. Because Raman probe and $\mathrm{pH}$ probe differed in spot size and shape (Raman probe: $0.3-\mathrm{mm}$ diameter, $\mathrm{pH}$ probe: $10 \mathrm{~mm} \times 0.2 \mathrm{~mm}$ ), the exact registration between Raman and $\mathrm{pH}$ measurement spots was not possible. Instead, the measurement spots of the transmission spectra overlapped with those of the Raman spectra. As shown in Fig. 3, each tissue sample was laid on the pHsensitive region. Then, a white light (Fiber-Lite DC950, DolanJenner) passed through the $\mathrm{pH}$-sensitive region and the transmission spectra varying according to the $\mathrm{pH}$ levels of the tissue samples were measured. During the $\mathrm{pH}$ experiments, the $\mathrm{pH}$ sensitive region was fixed onto a slide glass to avoid its undesired bending. Twenty transmission spectra from two different positions were measured for each breast tissue sample. All transmission spectra were acquired in the range of 640 to $760 \mathrm{~nm}$ with the spectral resolution of $0.08 \mathrm{~nm}$ and the acquisition time of $1 \mathrm{~s}$.

\subsection{Multivariate Statistical Analysis}

After the measurements of Raman spectra from the breast tissue samples, the instrument and CCD noise spectra, which were

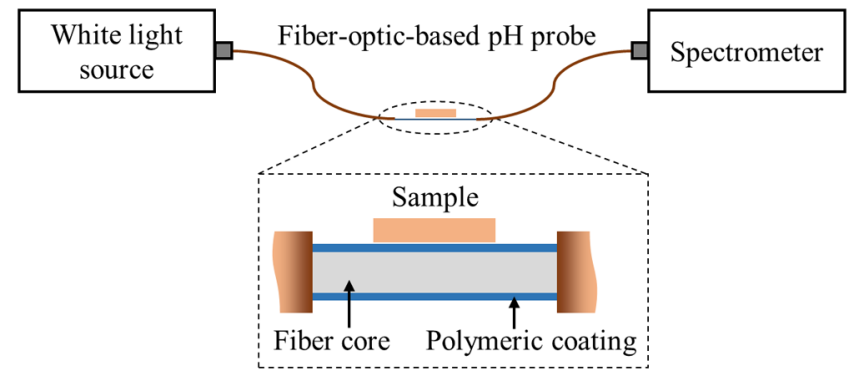

Fig. 3 Experimental setup using the fiber-optic-based $\mathrm{pH}$ probe for measuring the transmission spectra varying according to the $\mathrm{pH}$ level of the normal and cancerous breast tissue samples. The intensity of transmission spectra varies due to the absorbance change of the polymeric coating layer, which consists of NR/PAA bilayers.

measured without the samples were simply subtracted from the raw Raman spectra of the samples. The noise-corrected Raman spectra were smoothed by the Savitzky-Golay digital filter with smoothing width of 9 and degree of 3 followed by baselining using improved modified multipolynomial fitting. ${ }^{13,15}$ To remove the spectral variations by the fluctuations of excitation source, environment, and/or sample conditions, which are not related to the actual differences in the samples, the baseline-corrected Raman spectra were normalized by the vector normalization technique, ${ }^{29}$ where each of the Raman intensities corresponding to a Raman shift is divided by the Euclidean norm. For a dual-modal approach in multivariate statistical analysis, each normalized Raman spectrum was combined with each transmission spectrum obtained from the same sample. The combined spectra ( $\mathrm{pH}$-Raman spectra) have the wavelength range of 640 to $760 \mathrm{~nm}$ for the transmission spectra and 824 to $914 \mathrm{~nm}$ for the Raman spectra.

The 400 normalized Raman spectra (200 from cancerous samples and 200 from normal samples) were analyzed to evaluate the classification capability of only Raman spectroscopy. Whereas the $400 \mathrm{pH}-\mathrm{Raman}$ spectra (also 200 from cancerous samples and 200 from normal samples) were analyzed for the performance evaluation of the proposed dual-modal cancer detection based on optical $\mathrm{pH}$ sensing and Raman spectroscopy. PC-LDA was employed for the classification analysis on only Raman spectra and the pH-Raman spectra. LOOCV was used for evaluating the performance of the classification technique. All analyses were implemented using $\mathrm{R}$ program version 3.3.2.

\section{Results and Discussion}

Figure 4(a) shows the normalized and averaged Raman spectra for the normal and cancerous breast tissue samples. Each Raman spectrum represents the average (solid and dashed lines) and standard deviation (filled areas) calculated from 200 Raman spectra. The spectral profile is consistent with earlier studies, for example, phenylalanine ring breathing $\left(1009 \mathrm{~cm}^{-1}\right)$, phospholipids $\left(1080 \mathrm{~cm}^{-1}\right)$, amide III in proteins $\left(1270 \mathrm{~cm}^{-1}\right)$, $\mathrm{CH}_{2}$ deformation in lipids (1305 and $\left.1443 \mathrm{~cm}^{-1}\right)$, amide I in proteins $\left(1658 \mathrm{~cm}^{-1}\right)$, and carbonyl stretch $\left(1750 \mathrm{~cm}^{-1}\right){ }^{30-33}$ There exists spectral regions where the classification between normal and cancerous samples is possible with the naked eye. For the Raman spectra of the cancerous samples, it is observed that significant decrease in the normalized Raman intensity takes place in most of the spectral regions. In particular, the peak intensity at $1750 \mathrm{~cm}^{-1}$, usually due to fat content, ${ }^{33}$ ceases to exist in the cancerous sample case. Also, the peak 

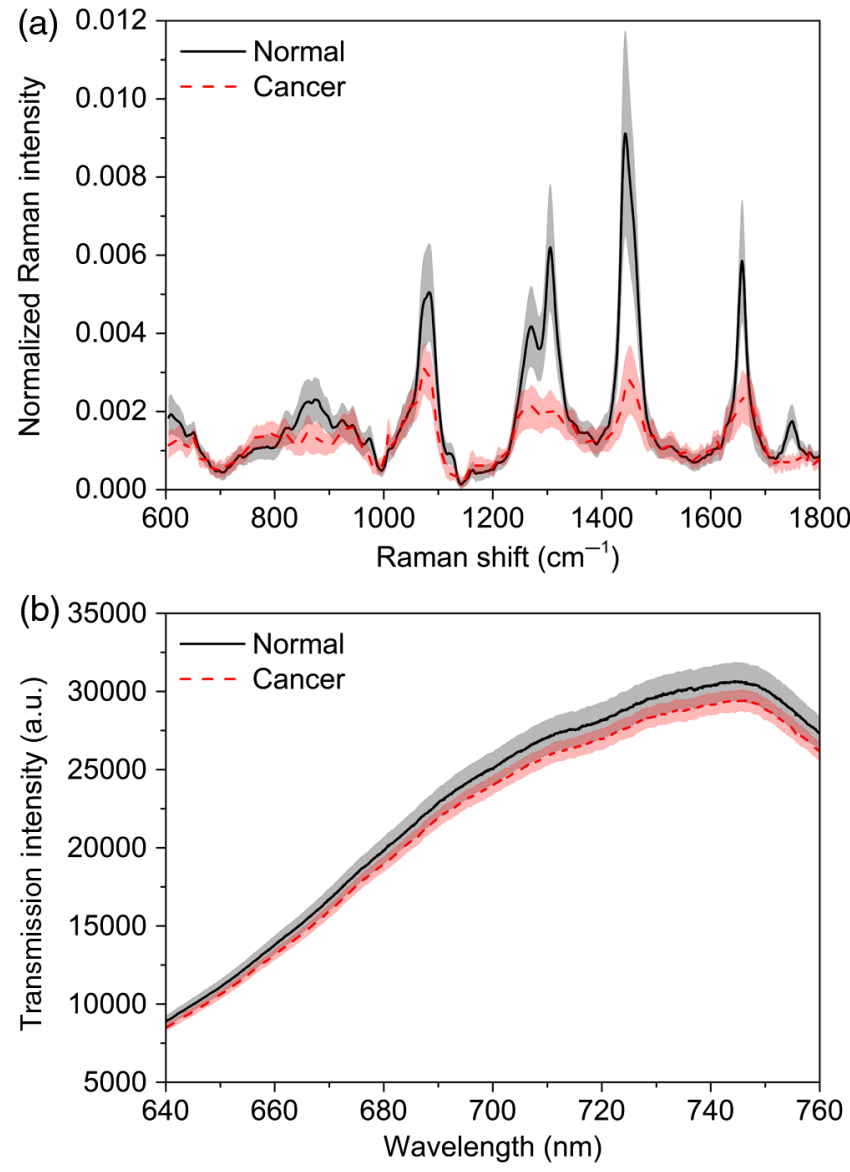

Fig. 4 (a) Normalized and averaged Raman spectra and (b) averaged transmission spectra, which were obtained from the normal and cancerous breast tissue samples (solid and dashed lines: average and filled areas: standard deviation). The Raman spectra were normalized by the vector normalization technique in which each of the Raman intensities corresponding to a Raman shift is divided by the Euclidean norm.

intensities at 1305 and $1443 \mathrm{~cm}^{-1}$ assigned to vibrational modes of lipids ${ }^{30-33}$ show much greater decrease than those at 1270 and $1658 \mathrm{~cm}^{-1}$ assigned to vibrational modes of proteins. ${ }^{30-33}$ That is to say that the intensity ratio of lipid to protein content is decreased for the cancerous sample case. On the other hand, the peak intensity at $1009 \mathrm{~cm}^{-1}$, usually related to phenylalanine ${ }^{32,33}$ which is essential amino acid of proteins, is stronger in the cancerous sample than in the normal one. The observed differences in Raman spectra between normal and cancerous samples can be thus understood to imply that normal breast tissue predominantly contains lipids, whereas cancerous breast tissue mostly contains proteins, which agrees with earlier study. ${ }^{30,32,33}$

Figure 4(b) shows the average (solid and dashed lines) and standard deviation (filled areas) of transmission spectra ( $n=200)$ obtained from the normal and cancerous breast tissue samples. For the whole transmission spectral region, it is observed that the average transmission intensities of normal samples are stronger than those of cancerous samples. These observations can be explained through the absorbance change of NR/PAA bilayer at varying $\mathrm{pH}$ level. Goicoechea et al. ${ }^{28}$ reported that the absorbance of NR/PAA bilayer decreases as the $\mathrm{pH}$ level is raised within the wavelength range from 600 to $800 \mathrm{~nm}$. This, in other words, means that the transmission

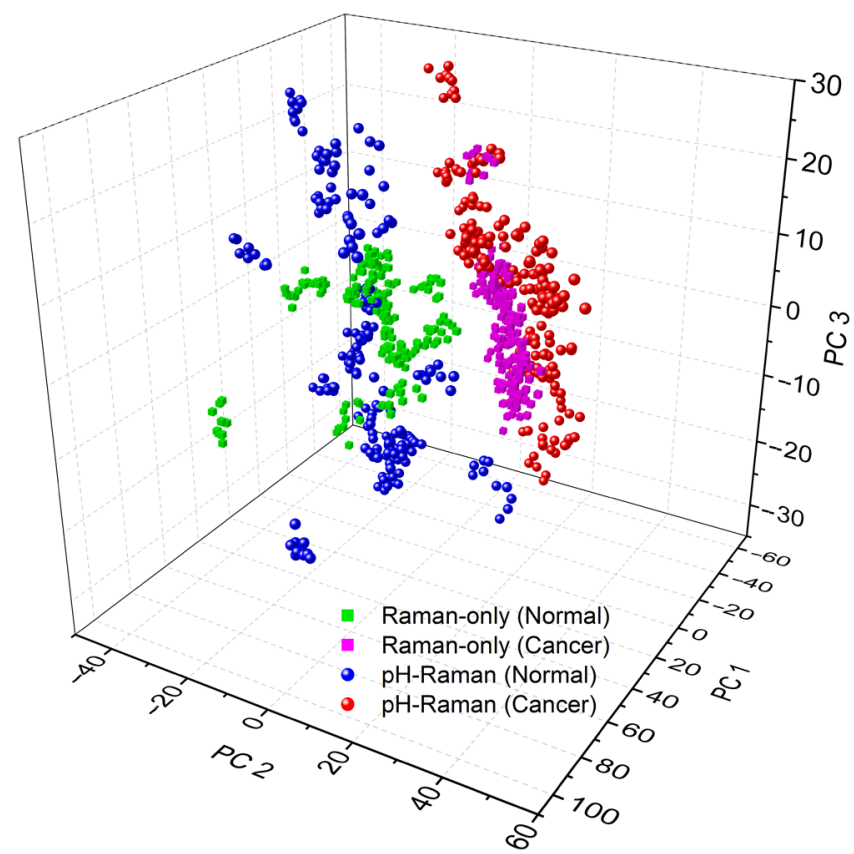

Fig. 5 Cluster plot showing PCA scores analyzed using Raman-only and $\mathrm{pH}$-Raman spectra of the normal and cancerous breast tissue samples. Percentage of variance explained: PC1 (82.7\%), PC2 (2.6\%), and PC3 $(2.1 \%)$ for Raman-only case/PC1 (73.5\%), PC2 (7.4\%), and PC3 (4.7\%) for pH-Raman case.

intensity by NR/PAA bilayer increases with increasing $\mathrm{pH}$ level. Therefore, from Fig. 4(b), it is confidently expected that the $\mathrm{pH}$ level of normal breast tissue samples is higher than that of cancerous ones. The integrated transmission intensity values of normal and cancerous samples were $4.335 \times 10^{7} \pm 0.176 \times$ $10^{7}$ and $4.133 \times 10^{7} \pm 0.12 \times 10^{7}$, respectively. Using the equation of $\mathrm{pH}$ calibration curve of Fig. 2(b), the $\mathrm{pH}$ levels of normal and cancerous samples were estimated to be about $7.37 \pm 0.9$ and $6.34 \pm 0.62$, respectively, which corresponds to the wellknown fact that the $\mathrm{pH}$ level of cancerous tissues is lower than that of normal tissues and lies within the $\mathrm{pH}$ range from 5.5 to $6.5^{20-22}$

Principal component analysis (PCA) was used to reduce the high dimensionality in the spectral data of the normal and cancerous samples. Figure 5 shows the cluster plot of PCA scores on the principal components (PCs) of normal and cancerous samples in Raman-only and pH-Raman spectral analysis. The first, second, and third PCs in Raman-only spectral analysis accounted for $82.7 \%, 2.6 \%$, and $2.1 \%$ of the total variance, respectively, whereas those in $\mathrm{pH}$-Raman spectral analysis were $73.5 \%, 7.4 \%$, and $4.7 \%$. In Fig. 5, it seems that PCA can successfully separate the cluster groups between normal and cancerous samples regardless of the use of $\mathrm{pH}$ data.

To more accurately assess the classification capability between the Raman-only and pH-Raman detection method, PC-LDA followed by LOOCV was used. The number of PCs is determined using two selection criteria: scree plot test and cumulative percentage variance. ${ }^{34}$ Figure 6 shows the scree plot of the eigenvalues (variances) along the $\mathrm{PC}$ number in Raman-only and pH-Raman spectral analysis. The scree plot test searches for a knee point in the plot curve where the optimal number of PCs reached. ${ }^{34}$ From the Raman-only curve in Fig. 6, the third PC can be selected as a knee point for Raman-only 


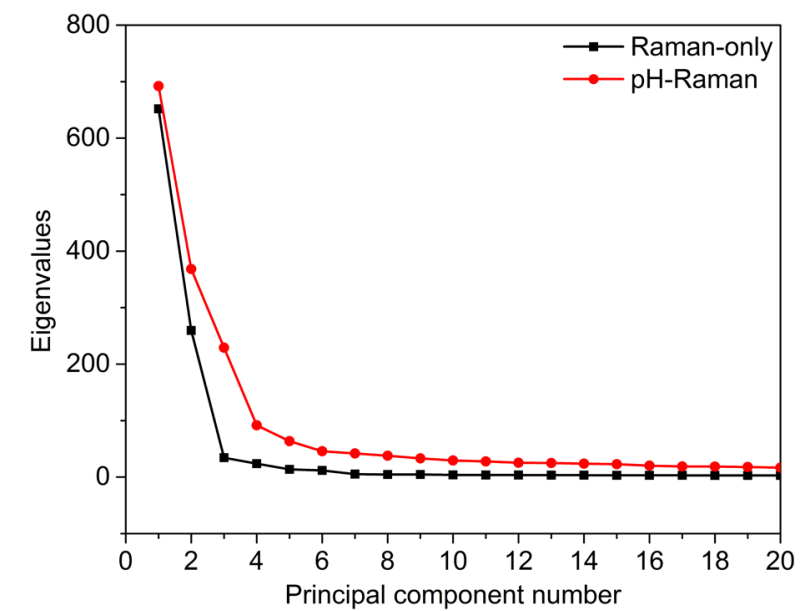

Fig. 6 Scree plot of the eigenvalues along the principal component number analyzed using Raman-only and $\mathrm{pH}$-Raman spectra of the normal and cancerous breast tissue samples. The scree plot test is used to determine the optimal number of PCs applied to the multivariate statistical analysis.

Table 1 Confusion matrix of Raman-only, pH-only, and pH-Raman spectral analysis.

\begin{tabular}{|c|c|c|c|c|c|c|}
\hline & \multicolumn{2}{|c|}{ Raman-only } & \multicolumn{2}{|c|}{ pH-only } & \multicolumn{2}{|c|}{ pH-Raman } \\
\hline & $\begin{array}{l}\text { Cancer } \\
\text { (actual) }\end{array}$ & $\begin{array}{l}\text { Normal } \\
\text { (actual) }\end{array}$ & $\begin{array}{l}\text { Cancer } \\
\text { (actual) }\end{array}$ & $\begin{array}{l}\text { Normal } \\
\text { (actual) }\end{array}$ & $\begin{array}{l}\text { Cancer } \\
\text { (actual) }\end{array}$ & $\begin{array}{l}\text { Normal } \\
\text { (actual) }\end{array}$ \\
\hline $\begin{array}{l}\text { Cancer } \\
\text { (predicted) }\end{array}$ & 200 (TP) & $17(\mathrm{FP})$ & $162(\mathrm{TP})$ & $34(\mathrm{FP})$ & 200 (TP) & $4(\mathrm{FP})$ \\
\hline $\begin{array}{l}\text { Normal } \\
\text { (predicted) }\end{array}$ & $0(\mathrm{FN})$ & $183(\mathrm{TN})$ & $38(\mathrm{FN})$ & $166(\mathrm{TN})$ & $0(\mathrm{FN})$ & $196(\mathrm{TN})$ \\
\hline
\end{tabular}

Note: TP, true positive; TN, true negative; FP, false positive; and FN, false negative.

spectral analysis. On the contrary, for $\mathrm{pH}-\mathrm{Raman}$ spectral analysis, it is difficult to determine the knee point due to the smooth curve. In cumulative percentage variance, the optimal number of PCs is determined using a user-defined threshold value of cumulative percentage of the variance (usually $90 \%$ to $99 \%$ of the variance) ${ }^{34}$ In this study, $90 \%$ was used as the threshold value. When the number of PCs was 5 (PC 1 to 5), the cumulative percentage of the variance was $90.5 \%$ and $90.4 \%$ for Raman-only and pH-Raman spectral analysis, respectively. Therefore, the PC 1 to 5 was used to perform classification analysis using PC-LDA with LOOCV.

The results of multivariate statistical analysis using the PC 1 to 5 are represented in Table 1 . The confusion matrix shows the tabular data for predicted class versus actual class of normal and cancerous samples in Raman-only, pH-only, and pH-Raman spectral analysis. The results of sensitivity $[=\mathrm{TP} /(\mathrm{TP}+\mathrm{FN})]$, true positive rate, for Raman-only and $\mathrm{pH}$-Raman spectral analyses are equal to 1 . On the other hand, the specificity $[=\mathrm{TN} /(\mathrm{TN}+\mathrm{FP})]$, true negative rate, are improved from 0.91 to 0.98 for $\mathrm{pH}$-Raman case. Enhanced specificity quantitatively presents the potential of dual-modal cancer detection using fiber-optic-based Raman and $\mathrm{pH}$ probes for accurate diagnosis.

\section{Conclusion}

From the experimental study of measuring the Raman spectra and $\mathrm{pH}$ levels of normal and cancerous breast tissue samples, it was found that both Raman spectra with chemical information and transmission spectra with $\mathrm{pH}$ information are useful in classifying normal and cancerous samples. When the dual-modal method based on Raman spectroscopy and optical $\mathrm{pH}$ sensing and multivariate statistical analysis work together, it is demonstrated that classification between normal and cancerous breast tissue samples was significantly improved. More specifically, multivariate statistical analysis using PC-LDA followed by LOOCV showed an enhanced specificity for breast cancer diagnosis. The suggested approach supports the potential practical applications of a pH-Raman-based detection method for breast cancer diagnosis.

\section{Disclosures}

All authors declare no conflicts of interest or financial relationships to disclose.

\section{Acknowledgments}

This work was supported by the National Research Foundation of Korea (NRF) grant funded by the Korean Government (2015M2A2A7A03043177 and 2017R1A2B4012428).

\section{References}

1. E. Vargis et al., "Effect of normal variations on disease classification of Raman spectra from cervical tissue," Analyst 136(14), 2981-2987 (2011).

2. S. Feng et al., "Blood plasma surface-enhanced Raman spectroscopy for non-invasive optical detection of cervical cancer," Analyst 138(14), 3967-3974 (2013).

3. R. Shaikh et al., "In vivo Raman spectroscopy of human uterine cervix: exploring the utility of vagina as an internal control," J. Biomed. Opt. 19(8), 087001 (2014).

4. M. Gniadecka et al., "Melanoma diagnosis by Raman spectroscopy and neural networks: structure alterations in proteins and lipids in intact cancer tissue," J. Invest. Dermatol. 122(2), 443-449 (2004).

5. C. A. Lieber et al., "Raman microspectroscopy for skin cancer detection in vitro," J. Biomed. Opt. 13(2), 024013 (2008).

6. H. Lui et al., "Real-time Raman spectroscopy for in vivo skin cancer diagnosis," Cancer Res. 72(10), 2491-2500 (2012).

7. I. P. Santos et al., "Raman spectroscopic characterization of melanoma and benign melanocytic lesions suspected of melanoma using highwavenumber Raman spectroscopy," Anal. Chem. 88(15), 7683-7688 (2016).

8. H. Abramczyk et al., "Raman imaging in biochemical and biomedical applications. Diagnosis and treatment of breast cancer," Chem. Rev. 113(8), 5766-5781 (2013).

9. B. Brozek-Pluska et al., "Raman spectroscopy and imaging: applications in human breast cancer diagnosis," Analyst 137(16), 37733780 (2012).

10. H. Krishna et al., "In vivo Raman spectroscopy for detection of oral neoplasia: a pilot clinical study," J. Biophotonics 7(9), 690-702 (2014).

11. M. S. Bergholt et al., "Fiberoptic confocal Raman spectroscopy for realtime in vivo diagnosis of dysplasia in Barrett's esophagus," Gastroenterology 146(1), 27-32 (2014).

12. J. Wang et al., "Comparative study of the endoscope-based bevelled and volume fiber-optic Raman probes for optical diagnosis of gastric dysplasia in vivo at endoscopy," Anal. Bioanal. Chem. 407(27), 8303-8310 (2015).

13. J. Addis et al., "Raman spectroscopy of endoscopic colonic biopsies from patients with ulcerative colitis to identify mucosal inflammation and healing," Biomed. Opt. Express 7(5), 2022-2035 (2016). 
14. R. Buividas et al., "Statistically quantified measurement of an Alzheimer's marker by surface-enhanced Raman scattering," J. Biophotonics 8(7), 567-574 (2015).

15. J. Zhao et al., "Automated autofluorescence background subtraction algorithm for biomedical Raman spectroscopy," Appl. Spectrosc. 61(11), 1225-1232 (2007).

16. S. Guo et al., "Optimization of Raman-spectrum baseline correction in biological application," Analyst 141(8), 2396-2404 (2016).

17. P. C. Ashok et al., "Multi-modal approach using Raman spectroscopy and optical coherence tomography for the discrimination of colonic adenocarcinoma from normal colon," Biomed. Opt. Express 4(10), 2179-2186 (2013).

18. L. Lim et al., "Clinical study of noninvasive in vivo melanoma and nonmelanoma skin cancers using multimodal spectral diagnosis," J. Biomed. Opt. 19(11), 117003 (2014).

19. K. Kenny et al., "Integrated Raman microscopy and auto-fluorescence imaging for fast tumour diagnosis during cancer surgery," in Optical Tomography and Spectroscopy, paper JW4A-7, Optical Society of America (2016).

20. P. A. Schornack et al., "Contributions of cell metabolism and $\mathrm{H}^{+}$ diffusion to the acidic pH of tumors," Neoplasia 5(2), 135-145 (2003).

21. R. A. Gatenby et al., "Why do cancers have high aerobic glycolysis," Nat. Rev. Cancer 4(11), 891-899 (2004).

22. R. Negrini et al., "pH-responsive lyotropic liquid crystals and their potential therapeutic role in cancer treatment," Chem. Сommun. 51(30), 6671-6674 (2015).

23. C. M. Monoranu et al., "pH measurement as quality control on human post mortem brain tissue: a study of the BrainNet Europe consortium," Neuropathol. Appl. Neurobiol. 35(3), 329-337 (2009).

24. M. Agenant et al., "Clinical superficial Raman probe aimed for epithelial tumor detection: phantom model results," Biomed. Opt. Express 5(4), 1203-1216 (2014).

25. L. Cui et al., "Aluminium foil as a potential substrate for ATR-FTIR, transflection FTIR or Raman spectrochemical analysis of biological specimens," Anal. Methods 8(3), 481-487 (2016).

26. G. Decher, "Fuzzy nanoassemblies: toward layered polymeric multicomposites," Science 277(5330), 1232-1237 (1997).

27. J. Hodak et al., "Layer-by-layer self-assembly of glucose oxidase with a poly(allylamine)ferrocene redox mediator," Langmuir 13(10), 27082716 (1997).

28. J. Goicoechea et al., "Optical fiber $\mathrm{pH}$ sensors based on layer-by-layer electrostatic self-assembled neutral red," Sens. Actuator B Chem. 132(1), 305-311 (2008).

29. R. Gautam et al., "Review of multidimensional data processing approaches for Raman and infrared spectroscopy," EPJ Tech. Instrum. 2(1), 1-38 (2015).

30. M. V. P. Chowdary et al., "Discrimination of normal, benign, and malignant breast tissues by Raman spectroscopy," Biopolymers 83(5), 556569 (2006).

31. S. K. Majumder et al., "Comparison of autofluorescence, diffuse reflectance, and Raman spectroscopy for breast tissue discrimination," J. Biomed. Opt. 13(5), 054009 (2008).
32. M. D. Keller et al., "Spatially offset Raman spectroscopy of layered soft tissues," Opt. Lett. 34(7), 926-928 (2009).

33. M. D. Keller et al., "Development of a spatially offset Raman spectroscopy probe for breast tumor surgical margin evaluation," J. Biomed. Opt. 16(7), 077006 (2011).

34. E. Ly et al., "Combination of FTIR spectral imaging and chemometrics for tumour detection from paraffin-embedded biopsies," Analyst 133(2), 197-205 (2008)

Soogeun Kim received his MS and PhD degrees in mechatronics from Gwangju Institute of Science and Technology in 2007 and 2015, respectively. $\mathrm{He}$ is a research professor at Kyung Hee University, Republic of Korea. His research interests focus on laser-tissue interaction phenomena and their applications, and ongoing research includes Raman spectroscopy for cancer detection, fiber-opticbased Raman spectroscopy, and spectrum data analysis by neural networks.

Seung Ho Lee received his MS degree in biomedical engineering from the Department of Medical Engineering, Kyung Hee University, in 2013. Since 2013, he has studied biomechatronics and biosensors with biomedical engineering on a doctorate path in the Department of Medical Engineering, College of Medicine at Kyung Hee University.

Sun Young Min received her BS degree in medicine from Chungnam National University in 2003 and her MS and PhD degrees in medicine from Kyung Hee University in 2008 and 2011, respectively. She is an assistant professor at Kyung Hee University. Her current research interests include breast cancer, cancer pathology, and convergence technology research. She is a member of the Korean Breast Cancer Society.

Kyung Min Byun received his BS and MS degrees from the School of Electrical Engineering, Seoul National University, Seoul, Republic of Korea, and his PhD from Seoul National University in 2007. From July 2007 to February 2008, he worked as a visiting scientist in the Department of Biomedical Engineering, Cornell University, Ithaca, New York. Currently, he is working as an associate professor in the Department of Biomedical Engineering, Kyung Hee University, Yongin, Republic of Korea. His main research activities are theoretical and experimental studies on high-sensitivity plasmonic biosensors with metallic nanostructures, Raman spectroscopy, and minimally invasive neuromodulation technique based on optical and ultrasound stimulation.

Soo Yeol Lee received his MS and $\mathrm{PhD}$ degrees in electronics engineering from Korea Advanced Institute of Science and Technology, Seoul, Republic of Korea, in 1985 and 1989, respectively. He was in the Department of Biomedical Engineering, Konkuk University, Republic of Korea, from 1992 to 1999. In 1999, he joined the Department of Biomedical Engineering, Kyung Hee University, where he is currently the director of the Functional and Metabolic Imaging Research Center. His current research interests include MRI, CT, elastography, and medical image/signal processing. 